\title{
VASCULARIZAÇÃO ARTERIAL DO TESTÍCULO DE SUÍNOS
}

\author{
ARTERIAL BLOOD SUPPLY OF THE TESTICLE IN PIGS
}

Anfibal de Sant'anna MORFTTI'; Vicente BORELLI'; Gilson TONIOLLO

\begin{abstract}
RESUMO
Utilizando 30 pares de tcstículos de suínos, com idade compreendida entre 6 a 10 meses, procedentes do Campus Administrativo de Pirassununga, da Universidade de São Paulo e da Seara Industrial de Xanxerê S.A., Santa Catarina, estudou-sc a vascularizaçăo artcrial mediante análise de moldes da artéria testicular, obtidos pela injeção de vynil. Na vascularização do órgão, a artéria testicular forneceu: (a) em 50,0\% das preparaçðcs, número variável de ramos laterais e mediais, ocorrendo $\mathrm{cm}$ $31,7 \%$, predominância dos ramos laterais; (b) $\mathrm{cm} 43,3 \%$ um ramo medial c outro lateral, havendo $\mathrm{cm}$ $30 \%$ das peças, predomínio do ramo medial; (c) em 6,7\% dos moldes, os ramos ventral c dorsal, notando-se predomínio do ramo ventral, na vascularização do órgåo. Nas preparaçðes $\mathrm{cm}$ que a artéria testicular fornecia númcro variável de ramos latcrais c mediais, observou-sc $\mathrm{cm}$ mediana $\mathrm{c}$ nos quadrantes dorsolateral, dorsomedial, ventrolateral e ventromedial, respectivamente, 27,0; 26,0;38,0; 28,0 vasos penctrantes quanto da predominância dos laterais c 24,$0 ; 24,0 ; 30,0$ c 33,0 no caso do predomínio dos mediais. Quando a artéria testicular cedcu dois ramos, o medial c o lateral, os valores foram, respectivamente, 25,$0 ; 24,0 ; 35,0 ; 28,0$ com predominância do medial c de 33,0; 33,5; 38,0; 37,5 com predominância do latcral. Quando cmitiu os ramos dorsal c ventral, verificou-se, respectivamente 30,5; 24,5; 26,5 c 28,0 quando da predominância do ventral. Observou-se cm 30 preparaçð̌es (50\%) ramos laterais ou mediais cedendo vasos penetrantes a quadrantes opostos ao destino principal do vaso que lhe deu origem. Foi verificada a presença de ramos adicionais, específicos, originados da artéria testicular antes desta ceder os ramos lateral c medial, e os ramos ventral e dorsal. Foram identificadas $\mathrm{cm}$ 95\% das preparaçðes, anastomoses entre a artéria testicular e seus ramos principais c colatcrais, bem como dos ramos adicionais. A ocorrência se deu com maior freqüência na facecaudal do órgāo sob a albugínca c ainda entre ramos da artéria testicular c cpididimários. Não houve diferença significativa no número total de vasos penctrantes dos testículos direito e esquerdo, ao nível de $\mathrm{P}>0,05$.
\end{abstract}

UNITERMOS: Anatomia, suínos; Testículos; Artérias; Suínos

\section{INTRODUÇĀO}

O cstudo da vascularização dos testículos dos animais domésticos e silvestres, reveste-se de grande importância no campo da reprodução animal, uma vez que os conhecimentos morfológicos, particularmente os relacionados ao sistema vascular, são indispensáveis para o melhor entendimento dos fenômenos ligados à termorregulação testicular, espermatogênese c mesmo à funçăo hormonal.

As informaçōes contidas nos tratados de Anatomia sobre os aspectos anatômicos da vascularizaçăo do testículo nos animais domésticos são cscassas e genéricas c, na maioria das vezes, contemplam apcnas a espécic eqüina (BOSSI s.d.; MONGIARDINO ${ }^{20}$, 1903; LESBRE ${ }^{17}, 1923$; MANU $^{19}$, 1930; ELLENBERGER; BAUM ${ }^{6}$, 1932; BRUNI; ZIMMERL 4, 1947; SISSON; GROSMAN ${ }^{25}$, 1959; KOCH ${ }^{16}, 1965$; SCHWARZE; SCHRÖDER $\left.{ }^{24}, 1972\right)$.

Por outro lado, as pesquisas que tratam especificamente do suíno, descrevem diferentes aspectos da vascularizaçðo, constatando extensa ramificaçāo no órgão, com a divisão da artêria testicular cm dois ramos e destes partindo várias outras ramificações (HILL ${ }^{12}, 1905$; HASUMI ${ }^{10}, 1931$; HARRISON ${ }^{9}$ 1949; NUNEZ; GETTY' ${ }^{23}$, 1969; FEHLINGS ${ }^{7}$, 1976; HEINZE; PTAK "1, 1976; JÁNTOSOVICOVA;
JANTOSOVIC'15, 1980; BOTTCHER; LANGE33, 1987). Acrescem-se ainda, as descriçōes dos diferentes tipos de vascularização, com a bifurcaçăo da artéria testicular na porçāo cilíndrica do órgão, na margem epididimária, ou ainda ñ̄o havendo divisão da mesma (BEUTLER!' 1922; GOTZSCHEBAUCH ${ }^{8}, 1939$ ).

Convém salientar as citaçócs que relatam as anastomoses encontradas cntre a artéria testicular e os ramos cpididimários no polo cranial do órgão (JANTOSOVICOVA ${ }^{14}$, 1969; HEINZE; PTAK ${ }^{11}, 1976$; FEHLINGS ${ }^{7}, 1976$; JANTOSOVICOVA; JANTOSOVIC ${ }^{15}$, 1980; BOTTCHER; LANGE $^{3}, 1987$ ), c as que apresentam arranjos vasculares, bem como o número c distribuiçāo dos vasos penetrantes nos diferentes quadrantes dos testiculos (VISINTIN ${ }^{26}$, 1985; DE VUONO', 1986; LOPES $^{18}$, 1987).

Seguindo esta última linha de pesquisa relacionada à estrutura c arquitetura dos componentes do funículo espermático dos mamíferos e dos diferentes aspectos da vascularizaçāo arterial, foi proposto $\mathrm{cm}$ suínos examinar o comportamento da artéria testicular e de scus ramos, bem como o número $\mathrm{c}$ distribuição dos vasos penctrantes. 


\section{MATERIAL E MÉTODO}

Utilizamos 30 pares de testículos de suínos, com idade compreendida entre 6 e 10 meses, procedentes do Campus Administrativo de Pirassununga - USP c da Seara Industrial de Xanxcrê, Santa Catarina.

Após o abatc, retiramos $\mathrm{cm}$ conjunto os testículos, cpidídimos, funículos espermáticos, escroto c a pele da rcgiåo com as respectivas papilas mamárias, que foram acondicionados $\mathrm{cm}$ sacos plásticos e congclados. No descongclamento das peças, separamos os testículos com os respectivos cpidídimos e funículos espermáticos, envoltos na lâmina visceral da túnica vaginal, a custa de abertura na parede do cscroto, túnica espermática externa, cremastérica, cremástica interna c lâmina parictal da túnica vaginal.

A seguir, isolamos a artéria testicular no terço médio da região crânio medial do órgão, incisando para tanto, a albugínca, c uma vez canulada, injetamos, com auxílio de scringa plástica, cerca de $10 \mathrm{ml} \mathrm{de} \mathrm{"vynil"} \mathrm{(VMCHB} \mathrm{-} \mathrm{1099)*} \mathrm{corado} \mathrm{cm}$ vermelho (Laca Nitrocelulose - vermelho molibdato)**. O material preparado foi mantido $\mathrm{cm}$ água corrente durante cerca de 1 hora $\mathrm{c}$ depois $\mathrm{cm}$ solução de ácido sulfúrico a $30 \%$, durante 71 a 96 horas, para obtenção dos moldes da vascularização anterial do órgão.

Para análise c descrição dos resultados, utilizamos desenhos esquemáticos das preparaçðes mostrando a divisăo da artéria testicular e seus ramos, bem como a origem e localizaçăo dos vasos penetrantes em relação aos quadrantes dorsolatcral, dorsomedial, ventrolatcral $\mathrm{c}$ ventromedial obtidos mediante traçado de dois planos perpendiculares entre si, sendo um de pólo a pólo, delimitando as metades lateral c medial do órgão, e outro no centro gcomécrico desse, demarcando as metades dorsal e ventral (Quad. 1).

Para estudo cstatístico, efetuamos o cálculo da mediana, correspondente ao númcro e distribuição dos vasos penetrantes, utilizando para comparar os valores, o teste de WILCOXON, segundo HOLLANDER; WOLF $^{13}$ (1973), adotando o nível de signifícância de $5 \%$.

\section{RESULTADOS}

Analisando os moldes da vascularização artcrial, verificamos que a artéria testicular alcança a superfícic do órgão cm correspondência ao seu pólo ventral, caminha $\mathrm{cm}$ trajeto sinuoso sob a albugínca $\mathrm{cm}$ direção ao pólo dorsal, sendo recoberta inicialmente pela cabeça do cpidídimo, para, mais comumente, antes de atingir este pólo, após contomá-lo, ou mesmo na altura dele, fornecer um ramo lateral e outro medial, ou ainda número variável de ramos laterais $\mathrm{c}$ mediais. $\mathrm{Em}$ alguns casos, quando na face medial, a artéria testicular ccede um ramo dorsal c outro ventral, apresentando assim, cinco arranjos vasculares (Grupos A, B, C, D c E) representados $\mathrm{cm} 60$ desenhos (MORETTR', 1988) c apresentado nesse trabalho pela observaçæo 19E c Figs. 1, 2, 3 c 4. A artéria testicular, no grupo A, (31,6\%), antes de alcançar o pólo dorsal do testículo, ou após contorná-lo, emite númcro variável de ramos laterais $\mathrm{e}$ mediais, dos quais emergem diretamente ou a partir de seus colatcrais, vasos penctrantes, de forma a existir nestes casos predominância dos ramos laterais na vascularizaçåo do órgāo (Fig. 1). No grupo B, (18,3\%), a artéria testicular, antes ou após contornar o pólo dorsal do testículo, ou a nível desse, cede número variável de ramos laterais c mediais, os quais cmitem dirctamentc ou a partir de scus colatcrais os vasos penctrantes, havendo nesses casos predominância de ramos mediais.

No grupo C, (30\%), a anćria testicular, antes de contornar o pólo dorsal do testículo, ou após ultrapassá-lo, divide-se nos ramos medial c latcral, de forma a existir nesses casos predominância do ramo mcdial (Fig. 2).

No grupo D $(13,3 \%)$, a artéria testicular, antes de contornar o pólo dorsal do testículo, após ultrapassá-lo, c ao nível desse, fornece os ramos lateral $\mathrm{c}$ medial, dos quais emergem a partir de seus colaterais número variável de vasos penetrantes, de tal forma a cxistir nesse grupo a predominância do ramo latcral (Fig. 3).

No grupo E $(6,7 \%)$, a artéria testicular alcança o órgão pela face medial, $\mathrm{c}$ no quadrantc dorsocaudal ou cranial, divide-se $\mathrm{cm}$ dois ramos, o dorsal $\mathrm{c}$ o ventral, existindo $\mathrm{cm}$ todos os casos predominância do ramo ventral (Fig. 4).

\section{Distribuiçāo e número de vasos penetrantes (V.P.)}

\section{Grupo A}

Obscrvamos $\mathrm{cm}$ mediana 120,0 (V.P.), cmitindo os ramos latcrais e scus colatcrais $72,0 \mathrm{c}$ os mediais c seus colatcrais 52,0 , distribuídos nas faces cranial c caudal do testículo.

Quanto à distribuição, encontramos cm medianas os scguintes valores nos quadrantes: dorsolateral 27,0; dorsomedial 26,0; ventrolatcral 38,0; ventromedial 28,0; distribuídos nas faces cranial c caudal do órgão. Os quadrantes laterais apresentam 62,0 (V.P.) c os mediais 54,0 , nas faces cranial c caudal da glândula cm todos os casos.

\section{Grupo B}

Determinamos $\mathrm{cm}$ mediana 109,0 (V.P.), fornecendo os ramos mediais c scus colaterais 61,0 , e os latcrais e scus colatcrais 49,0, distribuídos nas faces cranial c caudal do órgão.

Quanto à distribuição nos quadrantes, verificamos cm medianas os seguintes valores: dorsolateral 24,0 ; dorsomedial 24,0 ; ventrolateral 30,0 e ventromedial 33,0, observados nas faces cranial c caudal do testículo. Os quadrantes latcrais mostram $53,0 \mathrm{c}$ os mediais 58,0 , tamberm arranjados nas faces cranial c caudal do testículo.

\section{Grupo C}

Registramos nesses casos, cm mediana 114,5 (V.P.) sendo que os colaterais do ramo medial fornecem 74,0 , enquanto os colaterais do ramo lateral emitem 36,0 , notados nas faces cranial c caudal do órgão.

Obscrvamos na distribuição dos quadrantes, $\mathrm{cm}$ mediana os scguintes valores: dorsolateral 25,0; dorsomedial 24,0 ; ventrolatcral $35,0 \mathrm{c}$ vetromedial 28,0 anotados nas faces cranial 
MORETTI, A.S.; BORELL, V.; TONIOLLO, G. Vascularizą̧io anerial do lestículo de suínos. Braz. J. vel. Res. anim. Scl., São Paulo, v.29, p. 345-352, 1992. Suplemento.

c caudal do órgão. Os quadrantes laterais apresentam $60,0 \mathrm{c}$ os mediais 56,0 , observados nas faces cranial c caudal da glândula.

\section{Grupo D}

Verificamos cm mediana 137,5 (V.P.), contribuindo os colatcrais do ramo lateral com $95,0 \mathrm{c}$ os colaterais do ramo medial com 45,0, distribuídos nas faces caudal c cranial.

Relativamente à distribuiçăo nos quadrantes, anotamos $\mathrm{cm}$ medianas os seguintes valores: dorsolateral 33,0; dorsomedial 33,5; ventrolateral $38,0 \mathrm{c}$ ventromedial 37,0 , verificados nas faces cranial c caudal do órgåo. Deste modo, os quadrantes laterais apresentam 72,5, e os mediais 71,5 , observados nas faces cranial e caudal da glândula.

\section{Grupo E}

Encontramos $\mathrm{cm}$ mediana 116,0 (V.P.), cedendo os colaterais do ramo ventral $70,0 \mathrm{e}$ os colatcrais do ramo dorsal 33,5, situados nas faces cranial c caudal do testículo. Anotamos $\mathrm{cm}$ medianas nos quadrantes, os seguintes valores: dorsolateral 30,5 ; dorsomedial 24,5 ; vetrolateral 26,5 e ventromedial 28,0 , distribuídos na face cranial c caudal do órgð̃o.

Desta mancira, os quadrantes laterais apresentam $56,6 \mathrm{c}$ os quadrantes mediais 54,5 situados na face cranial c caudal da glândula.

\section{Comportamento dos ramos da artéria testicular}

\section{Grupos A e B}

Identificamos a presença de vasos penetrantes oriundos de ramos laterais $\mathrm{c}$ scus colatcrais nos quadrantes mediais, o mesmo ocorrendo $\mathrm{cm}$ relaçăo aos ramos mediais c scus colaterais alcançando os quadrantes latcrais no grupo A. No grupo B, respectivamente, os ramos mediais ou laterais e seus colatcrais, tamberm alcançando os quadrantes laterais ou mcdiais. Da mesma mancira, destacamos o comportamento dos ramos laterais $\mathrm{c}$ seus colaterais que forncecm vasos penetrantes aos quadrantes mediais após terem percorrido dois quadrantes laterais caudal $\mathrm{c}$ cranial, para atingir um quadrante medial na sua face cranial. Os ramos mediais $\mathrm{c}$ scus colaterais também mostram comportamento similar. No grupo B também verificamos o mesmo, partindo, respectivamente, dos ramos mediais ou latcrais $\mathrm{c}$ scus colaterais.

\section{Grupos C e D}

Verificamos a presença de vasos penetrantes oriundos dos colatcrais do ramo medial nos quadrantes laterais $\mathrm{c}$ colatcrais do ramo lateral nos quadrantes mediais, o mesmo ocorrendo com o Grupo D, respectivamente, para os colaterais do ramo lateral ou medial, que atingem os quadrantes mediais ou laterais.

Também encontramos, no Grupo $C$, colaterais do ramo medial fornecendo vasos penetrantes aos quadrantes laterais, depois de terem percorrido dois quadrantes mediais, caudal c cranial, para atingir um quadrante tatcral. Os colaterais do ramo lateral, da mesma mancira, apresentam comportamento idêntico.
No grupo $\mathrm{D}$, também observamos o mesmo, partindo, respectivamente, dos colaterais do ramo lateral $\mathrm{c}$ medial.

Mencione-se ainda, o comportamento de colaterais do ramo medial (Grupo C), que emitem vasos penctrantes para o quadrante medial, porém, depois de percorrerem os dois quadrantes latcrais (caudal c cranial), para alcançarem um quadrante medial, na sua face cranial.

Regisure-se nessas observaçð̃es c nos Grupos C e D, originando-se da artéria testicular $\mathrm{e}$ antes da divisăo $\mathrm{cm}$ ramo medial e latcral, com predominância do primciro ou do scgundo, a ocorrencia de ramos adicionais que fornocem vasos penetrantes aos quadrantes medial c lateral.

\section{Grupo E}

Neste grupo verificamos os colaterais do ramo ventral cedendo número variável de vasos penctrantes aos quadrantes dorsais, $\mathrm{c}$ os colaterais do ramo dorsal somente uma vez. Originando-sc da artéria testicular, antes da divisło $\mathrm{cm}$ ramo ventral c dorsal, observamos a presença, cm duas peşas, de ramos adicionais.

Nos órgāos examinados, detcrminamos, cm medianas, independente do grupo considerado, 118,5 vasos penetrantes no testículo direito c 119,5 no esquerdo, nåo havendo diferença estatística significativa.

Identificamos, $\mathrm{cm} 95 \%$ das peças, anastomoses nas quais participam os vasos implicados na nutrição do testículo, enconurando maior frequiência na face caudal do órgão sob a albugínea c entre colaterais de um mesmo ramo ou de ramos diferentes. Evidenciamos também inúmeras anastomoses entre colaterais de ramos laterais ou mediais com vasos responsáveis pela vascularizaçăo do cpidídimo, sendo mais frequientes na face cranial dos quadrantes ventrolatcral c ventromedial.

\section{DISCUSSĀO}

Apcsar das diferentes designaçð̋es empregadas pelos autores quando cstudam a artéria responsável pela vascularizaçăo do testículo, utilizamos no presentc cstudo a denominação artéria testicular, que consta da NOMINA ANATÔMICA VETERINÁRIA $^{2}$ (1983). Adolamos para os vasos destinados ao parênquima testicular, oriundos da artéria testicular c scus colatcrais, a denominaçăo de vasos penctrantes, enquanto os termos ramos lateral e medial, reservamos às principais contribuiçðes oriundas diretamente da artéria testicular, c ainda, chamamos de ramo adicional, aos vasos que nascem desta artéria antes dela ceder os ramos lateral e medial, como já o fizeram, VISINTIN"26, (1985); DE VUONO', (1986) e LOPES $^{18}$, (1987).

Examinando os modclos de vynil, verificamos que nos suínos a artéria testicular penetra na túnica albugínea próximo ao polo ventral do órgåo, caminha com trajcto sinuoso $\mathrm{cm}$ direção ao polo dorsal, sendo recoberta inicialmente pela cabeça do cpidídimo, ocupando depois posiçāo medial cm relação ao scu corpo. Nos tratados de Anatomia Veterinária, cm razão dos autores considerarem a anatomia da cspécic cquilina como padrão, encontramos informaçðes divergentes quanto ao comportamento desse vaso. (BOSSI ${ }^{2}$ s.d.; MONGIARDINO ${ }^{20}$, 1903; LESBRE17, 1923; MANU19, 1930; ELLENBERGER; BAUM ${ }^{6}, 1932$; BRUNI; ZIMMERL 4 1947; SISSON; GROSMAN $\left.{ }^{3}, 1959\right)$. Em scu percurso, quando o polo dorsal 
MOREITI, A.S.; BORELJJ, V.; TONIOLLO, G. Vascularizaçăo arterial do testículo de suínos. Braz. J. vet. Res. anlm. Sci., Såo Paulo, v.29, p. 345-352, 1992. Suplemento.

do órgão caracterizando os cinco modelos já descritos, somente SCHWARZE; SCHRÖDER ${ }^{24}$, (1972), dentre os tratadistas, destacam genericamente uma distribuiçăo diferentc em cada espécie.

Informaçōes semelhantes às nossas colhemos em vários trabalhos especializados (HILL' ${ }^{12}, 1905$; BEUTLER', 1922; H A S UM I ${ }^{10}, 1931$; G OTZ S C HE B A U C H ${ }^{8}, 1939$; HARRISON ${ }^{9}, 1949$; NUNEZ; GETTY ${ }^{23}, 1969$; HEINZE; PTAK ${ }^{11}$,1976; FEHLINGS ${ }^{7}, 1976$; JANTOSOVICOVA; JANTOSOVIC'15, 1980; BOTTCHER; LANGE ${ }^{3}, 1987$ ).

Alguns destes autores fazem referência à divisão da artéria testicular e à possibilidade desta fomecer ramos mediais c latcrais (HILL'2, 1905; HASUMI ${ }^{10}, 1931$ c NUNEZ; GETTY $^{23}, 1969$ ), outros assinalam sua divisăo $\mathrm{cm}$ dois ramos destinados na maioria das vezes às faces lateral c medial (HARRISON', 1949; HEINZE; PTAK" ", 1976; FEHLINGS ${ }^{\prime}$, 1976; JANTOSOVICOVA; JANTOSOVIC's, 1980; BOTTCHER; LANGE ${ }^{3}, 1987$ ) c ainda alguns registram difcrentes arranjos de vascularização testicular (BEUTLER ${ }^{1}, 1922$ e GOTZSCHEBAUCH ${ }^{8}, 1939$ ). Todavia, nenhum deles caracteriza nos suínos, as diferentes freqüiencias das modalidades de vascularização $\mathrm{cm}$ função dos tipos de divisão c de distribuiçăo da artéria testicular, como destacamos no presentc estudo. Comparando os diferentes tipos de arranjos vasculares encontrados nos suínos, sua freqüência c tipos predominantes, com os trabalhos que utilizaram a mesma técnica, verificamos nos bovinos da raça Nclore (VISINTIN ${ }^{26}$, 1985) e nos taurinos (LOPES $\left.{ }^{18}, 1987\right)$, nos casos em que a artéria testicular cedia dois ramos, prcdomínio do ramo lateral sobrc o medial, enquanto no jumento nordestino (DE VUONCs, 1986), verifica-sc predomínio dos mediais sobre os latcrais, nos casos $\mathrm{cm}$ que a artéria testicular fornecia número variável dos dois ramos. Nos suinos não cvidenciamos cquivalência de participação dos ramos lateral c medial na vascularização, cncontrada nos zebuínos (VISINTIN²6, 1985), taurinos (LOPES ${ }^{18}, 1987$ ) e jumento nordestino (DE VUONO', 1986), não se verificando nestes o arranjo vascular correspondente ao grupo $\mathrm{E}$, onde a artéria testicular emite um ramo dorsal c outro ventral. Os autores (VISINTIN²6, 1985; DE VUONO ${ }^{3}, 1986$ c LOPES $\left.^{18}, 1987\right)$, não assinalaram casos dc ramos laterais ou mediais cedendo vasos penetrantes a quadrantes opostos ao vaso que lhe deu origem, como identificamos nos suínos, enquanto nas espécies estudadas, zebuínos, laurinos, jumento nordestino c suínos, evidenciamos que os ramos denominados de adicionais, ou complementares somente estiveram presentes nos taurinos.

$\mathrm{Na}$ análise dos arranjos vasculares encontrados nos suínos, notamos os mesmos achados no jumento nordestino (DE VUONO์, 1986) quando a artéria testicular fornecia número variável de ramos laterais c mediais, com predominância do primciro, ou scgundo (Grupos A c B), sendo tais modalidades de vascularização consideradas $\mathrm{cm}$ conjunto nos zebuínos (VISINTIN²6, 1985) e nos taurinos (LOPES ${ }^{18}$, 1987). No caso da artéria testicular ceder um ramo medial e outro lateral, com predominância do primciro ou do scgundo, tais variaçōes também foram descritas nos zcbuínos, taurinos e jumento nordestino, todavia com maior participação do ramo lateral, fato contrário do obscrvado nos suínos.

Quanto ao número total de vasos penetrantes, registramos nos suínos, como mediana 118,5 para o testículo direito c 119,5 para o testículo esquerdo. Nas outras espécies, jumento nordestino (DE VUONO', 1986), foram encontrados na mesma sequiência 86,5 no testículo direito c 86,0 no testículo esquerdo, no zebuíno (VISINTIN ${ }^{26}$, 1985) 41,5 para o dircito e 43,5 para o csquerdo, c nos taurinos (LOPES ${ }^{18}, 1987$ ) 38,5 para 0 testículo direito e 29,0 para o esquerdo, o que demonstra maior número desses vasos nos suínos, não ocorrendo diferença estatística significativa entre os valores das medianas do total de vasos dos testículos esquerdo c dircito, fato que ocorrcu tambćm nos taurinos (LOPES ${ }^{18}, 1987$ ).

As anastomoses que evidenciamos nos suínos, encontramos apenas nos taurinos (LOPES $\left.{ }^{18}, 1987\right), \mathrm{c} \mathrm{cm}$ número reduzido de casos. Também identificamos as anastomoses entre os ramos da artéria testicular c ramos cpididimários mencionados por JANTOSOVICOVA ${ }^{14}$ (1969); HEINZE; PTAK"'(1976); FEHLINGS $^{7}$ (1976); JANTOSOVICOVA; JANTOSOVIC ${ }^{15}$ (1980).

\section{CONCLUSŌES}

Do que acabamos de expor concluímos:

1. a artéria testicular, nos suínos, alcança a superfícic do órgão $\mathrm{cm}$ correspondência ao scu pólo ventral, direciona-se cm scguida para o pólo dorsal, caminhando sob a túnica albugínca com trajeto sinuoso, inicialmentc sob a cabcça do cpidídimo, c depois $\mathrm{cm}$ posição mcdial $\mathrm{cm}$ rclação ao corpo cpididimário, para após contorná-lo ou mesmo na altura delc, forneccr: (a) número variável de ramos laterais e mediais, com predominância do primciro ou do scgundo; (b) um ramo medial c outro lateral com predominância do primciro ou do segundo; (c) um ramo ventral c outro dorsal, com predominância do primciro;

2. quando a artéria testicular emite número variável de ramos laterais e mediais, com predominância dos ramos laterais, observa-se cm mediana 120,0 vasos penetrantes, sendo que os ramos laterais e seus colaterais fornecem 72,0 e os ramos mediais e scus colaterais 52,0 ;

3. quando a artéria testicular cede número variável de ramos latcrais c mediais, com predominância dos ramos mediais (18,3\%), registra-se como mediana 109,0 vasos penetrantes, onde os ramos mediais e seus colaterais cedem 61,0 e os ramos laterais e scus colaterais 49,0 ;

4. quando a artéria testicular fornece os ramos medial $c$ lateral, com predominância do ramo medial, verifica-se cm mediana 141,5 vasos penctrantes, contribuindo os colatcrais do ramo medial com $74,0 \mathrm{c}$ os colaterais do ramo lateral com 36,0;

5. quando a artéria testicular cmite os ramos medial $\mathrm{c}$ lateral com predominância do ramo lateral, nota-se cm mediana 137,5 vasos penetrantes, de modo que os colaterais do ramo lateral participam com $95,0 \mathrm{e}$ os colaterais do ramo medial com 45,0;

6. quando a artéria testicular emite os ramos ventral e dorsal com o predomínio do ramo ventral, registra-se cm mediana 116,0 vasos penctrantes, sendo que os colaterais do ramo ventral fornecem 70,0 c os colaterais do ramo dorsal 33,5;

7. analisando-se os valores das medianas nos testículos direitos, o quadrante ventromedial apresenta o maior número de vasos penctrantes $(33,0)$, seguido pelos quadrantes ventrolateral $(32,0)$, dorsolateral $(27,0)$ e dorsomedial $(25,5)$ enquanto nos testículos esquerdos concentram-se maior númcro de vasos penetrantes no 
quadrante ventrolateral $(38,0)$, seguido do quadrante ventromedial $(29,5)$, dorsolateral $(27,0)$ e dorsomedial $(26,0)$.

8. quando a artéria testicular cede número variável de ramos laterais c mediais, com predominância dos ramos laterais, observa-se presença de vasos penetrantes oriundos dos ramos laterais e scus colaterais, nos quadrantes mediais e de vasos penetrantes oriundos dos ramos mediais c seus colaterais nos quadrantes laterais. Comportamento semclhante obscrva-se quando há predominância dos ramos mediais;

9. quando a artéria testicular cede os ramos medial c lateral, com predominância do ramo medial, observa-sc ocorrência de vasos penctrantes, oriundos dos colaterais do ramo medial, nos quadrantes laterais e vasos penctrantes dos colatcrais do ramo lateral nos quadrantes mediais. Comportamento semelhante ocorre na predominância do ramo lateral;

10. quando a artéria testicular cede o ramo medial c o latcral, fornece antes ramos adicionais que cedem vasos penetrantcs aos quadrantes mediais e laterais, concentrados nos quadrantes mediais;

11. quando a artéria testicular cede o ramo ventral c o dorsal, emite antes ramo adicional que dá origem a vasos penctrantcs para o quadrantc dorsomedial;

12. anastomoses anteriais de que participam a artéria testicular, seus ramos e colaterais, bem como os colaterais de ramos adicionais, ocorrem $\mathrm{cm}$ quase todas as preparaç̋es $(95,0 \%)$ com maior freqüência na face caudal do órgāo, sob a túnica albugínca.

13. não existem diferenças estatisticamente significantes quando se compara os valores das medianas, referente ao total de vasos penetrantes dos testículos dircito e esquerdo;

14. nos arranjos vascularcs estudados quando a artéria testicular emitc número variável de ramos laterais $\mathrm{e}$ mediais, ou quando ela fornece apenas um ramo lateral c outro medial, ocorre a presença de vasos penetrantes $\mathrm{cm}$ quadrantes opostos ao vaso que lhe deu origem.

\section{SUMMARY}

The arterial blood supply of 30 pairs of swines testicles were studied. The animals 6 to 10 months old were obtained from the "Campus Administrativo de Pirassununga, Faculdade de Medicina Veterinária c Zootecnia - USP" and from "Scara Industrial de Xanxerê S/A - Santa Catarina". The testicular artery was injected with vinyl in order to obtain arterial models. In $50 \%$ of the preparations the testicular antery showed a variable number of lateral and medial branches; in $31.7 \%$ there was predominance of lateral branches. On the remaining $18.3 \%$ it was observed predominance of the medial branches. In $43.3 \%$ the testicular artery originated two branches, a lateral and a medial. In $30.0 \%$ of the preparations there was predominance of the medial branch, while the lateral one predominated on $13.3 \%$. On the remaining $6.7 \%$ of the organs the testicular antery gave a dorsal branch and a ventral branch, the later prevailing on the vascularization of the organ. Concerning to the number and distribution of the arterial penetrant vessels, as analysed by the median method, it was possible to observe little differences among quadrants on the various models studied. When the testicular artery originated a variable number of lateral and medial branches (50\%), it was possible to observe the presence of penetrant vessels that starting from the branching point reach oposite quadrants to the principal destination of the original artery. In $20.0 \%$ of the preparations when the division resulted in a lateral branch and a medial one, there were additional branches originating from the testicular artery prior to its division point. In 59 models (95.0\%) anastomosis between the testicular artery and its main branches of theirs collaterals were identificd. Those anastomosis were observed under thin albuginea and in the caudal face of the organ generally. Other anastomosis were noted between branches of the testicular and epididymary arteries. There were no significant differences among median values $(P>0,05)$ of total number of penetrant vessels of the right or the left testicles.

UNITERMS: Anatomy of swinc; Testis; Arterics; Swine

\section{REFERÊNCIAS BIBLIOGRÁFICAS}

01-BEUTLER, 1922 apud FEHLINGS, K.7. p. 10-1.

02-BOSSI, V. Angiologia. In: BOSSI, V.; CARADONNA, G.B.; SPAMPANI, G.; VARALDI, L.; ZIMMERL, U. Trattato dI anatomia veterinaria. Milano, Francesco Vallardi, s.d. v. 2, p. 210-3.

03-BOTTCHER, M.; LANGE, W. Untersuchungen am arteriellen Gefassystem des Eberhodens. Arch. exp. Vet. - Med., v.411, p. 58-64, 1987.

04-BRUNI, A.C.; ZIMMERL, U. Anatomia degli animall domesticl Milano, Francesco Vallardi, 1947. v.2, p. 540.

05-DE VUONO, L. Contribuiçāo ao estudo da vascularizaçāo arterial do testículo em jumento nordestino. São Paulo, 1986. Tese (Doutorado) - Faculdade de Medicina Veterinária e Zootecnia, Universidade de Säo Paulo.

06-ELLENBERGER, W.; BAUM, H. Handbuch der verglelchenden Anatomie der Haustiere. 17 Auf. Berlin, Julius Springer, 1932. p. 696.

07-FEHLINGS, K. Korrosions-und rontgenanatomische Untersuchungen der Arteria testicularis von Katze, Hund, Schwein, Schaf, Rind und Pferd. Hannover, 1976. (Inaugural Dissertation) - Tierarztliche Hochschule.

08-GOYTZSCHEBAUCH, A. Der Hoden der Haussaugetiere und seine Hullen in biologischer und artiagnostisher Hinsicht. $\mathbf{Z}$. Anat. Entwicklungsgesch., v 105, p. 433-58, 1939.

09-HARRISON, R. G. The comparative anatomy of the blood supply of the mammalian testis. Proc.Zool. Soc. Lond., v.119, p. 32547, 1949.

10-HASUMI, S. Anatomische Untersuchungen uber der Blutgefasse der Hoden und Nebenhoden von verschiedenen Saugetieren. Jap. J. med. Scl. Blol., v.2, p. 135-50, 1931

11-HENZE, W.; PTAK, W. Vergleichenden morphologische Untersuchugen am Blutgefass-system des Hodens von Rind, Schwcin, Pferd und Hund unter funktionellen Aspekten. Arch. exp. Vet. - Med., v.30, p. 669-85, 1976.

12-HILL, E. C. On the gross development and vascularization of the testis Amer. J. Anat., v.6, p. 439-59, 1905.

13-HOLLANDER, M.; WOLF, D. A. Nonparametric statistical methods. New York, John Wiley, 1973.

14-JANTOSOVICOVA, J. To the question of anastomoses of the arterics of ram, boar and stallion testis and epididymis. Folia Vet., Praha, v.13, p. $21-6,1969$. 
15-JANTOSOVICOVA, J.; JANTOSOVIC, J. Topographischanatomische Angaben uber A. testicularis, A. ducturdeferentis und A. cremasterica beim Eber. Gegenbaurs Morphol. Jahrb., v. 126, p. 756-65, 1980.

16-KOCH, T. Lehrbuch der Veterinar-Anatomle. Jena, Gustav Fihser, 1965. v.3, p. 124.

17-LESBRE, F.X. Précis d'anatomle comparée des anlmaux domestiques. Paris, J. B. Baillière, 1923. v.2, p. 336-43.

18-LOPES, P.R.B. Contribuição ao estudo da vascularização arterial do testículo em bovlnos (Bos tauros). Sāo Paulo, 1987. Tese (Doutorado) - Faculdade de Medicina Veterinária e Zootecnia, Universidade de São Paulo.

19-MANNU, A. Apparechio vascolare. In: ZIMMERL, U. Trattato di anatomla veterinarla. Milano, Francesco Vallardi, 1930. v.2, p. $170-2$.

20-MONGIARDINO, T. Trattato dl anatomia topograflca del mammiferi domesticl. Torino, Luigi Delgrosso, 1903.

21-MORETTI, A. S. Contribuiçāo ao estudo da vascularizaçāo arterlal do testículo de suínos. São Paulo, 1988. Tese
(Doutorado) - Faculdade de Medicina Veterinária e Zootecnia, Universidade de São Paulo.

22-NOMINA ANATOMICA VETERINARIA. 3 ed. Ithaca, International Committee on Veterinary Gross Anatomical Nomenclature, 1983.

23-NUNEZ, Q.; GETTY, R. Arterial suppy to the genitalia and acessory genital organs of swine. Iowa St. J. Scl., v.44, p. 93127, 1969.

24-SCHWARZE, E.; SCHRODER, L. Compendlo de anatomia veterinárla e aparato circulatorio y plel.Zaragoza, Acribia, 1972. v.3, p. 72-3.

25-SISSON, S.; GROSSMAN, J. D. Anatomia de los animales domesticos. 4 ed., Barcelona, Salvat, 1959. p. 658-706.

26-VISINTIN, J. A. Contribulçāo ao estudo da vascularlzação arterial do testículo, em bovinos da raça Nelore. São Paulo, 1985. Tese (Doutorado). - Instituto de Ciências Biomédicas, Universidade de São Paulo.

Recebido para publicação $\mathrm{cm} 21 / 11 / 91$ Aprovado para publicação em12/08/92 
Representação fotográfica (Fig. 1, 2, 3 e 4), da vascularização arterial do testículo de suínos obtido em modelos de "vinyl" correspondentes aos grupos A, B, C, D e E.

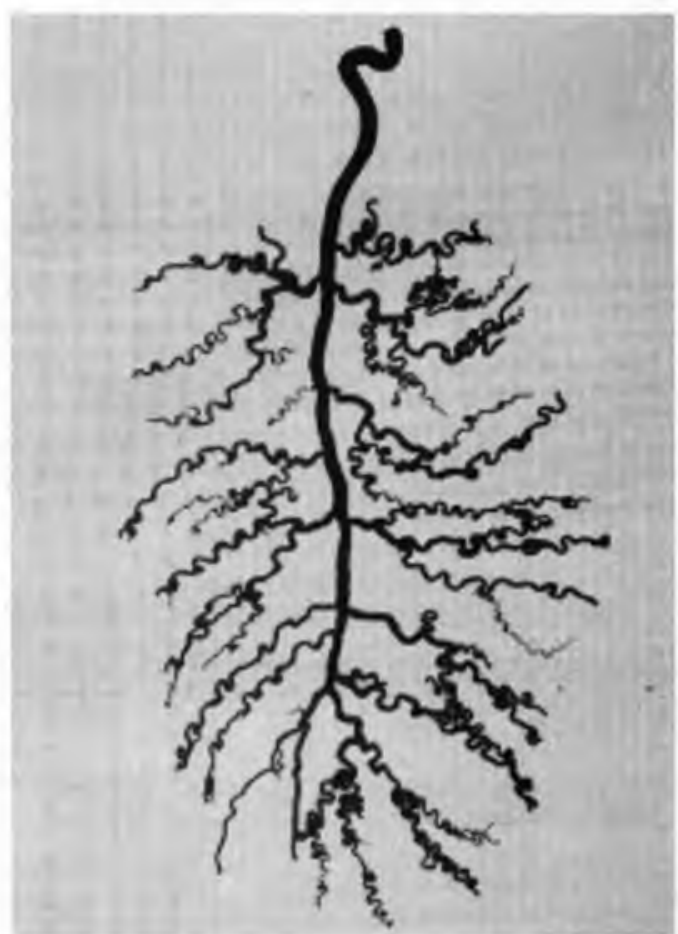

Figura 1

(Testículo direito)

a - artéria testicular

$\mathrm{b}$ - ramos laterais

c - ramos mediais

d - colaterais

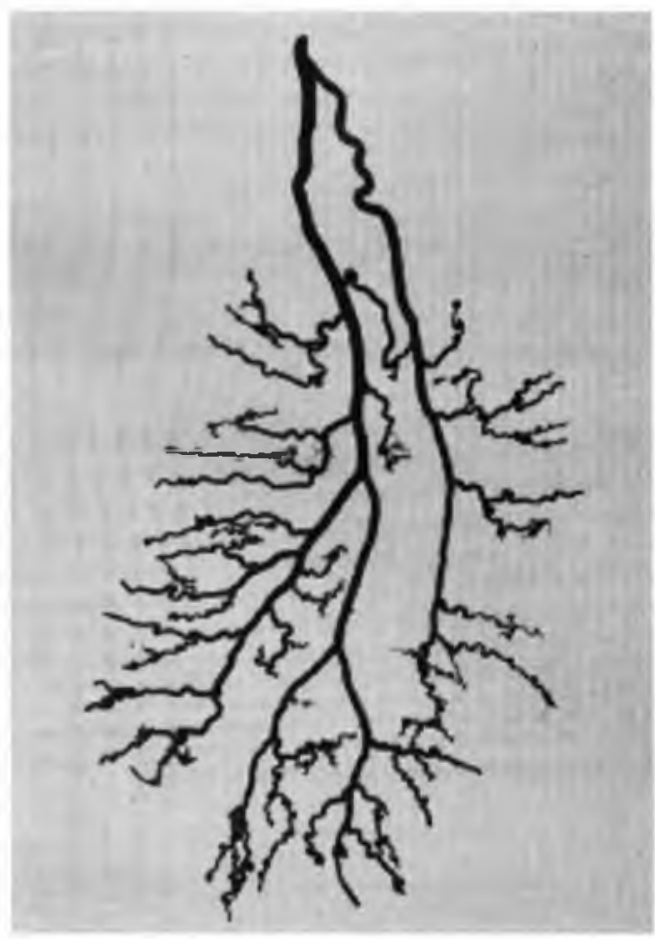

(Testículo esquerdo)

a - artéria testicular

b - ramo medial

c - ramo lateral

$\mathrm{d}$ - colaterais

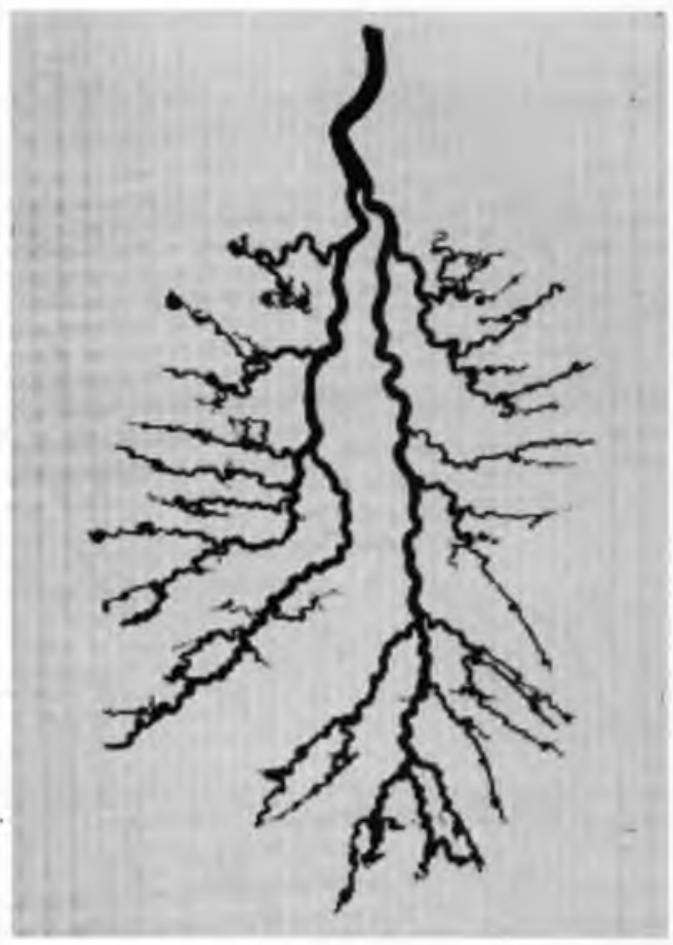

(Testículo esquerdo)

a - artéria testicular

b - ramo medial

c - ramo lateral

$d$ - colaterais

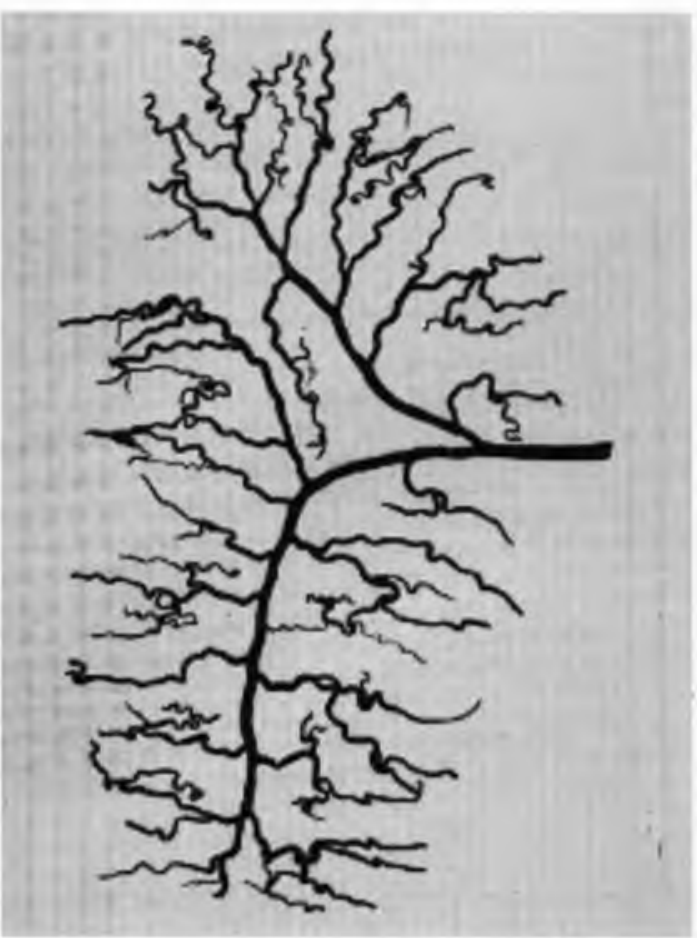

(Testículo esquerdo)

Figura 4

- anteria testicular

b - ramo dorsal

c - ramo ventral

d - colaterais 


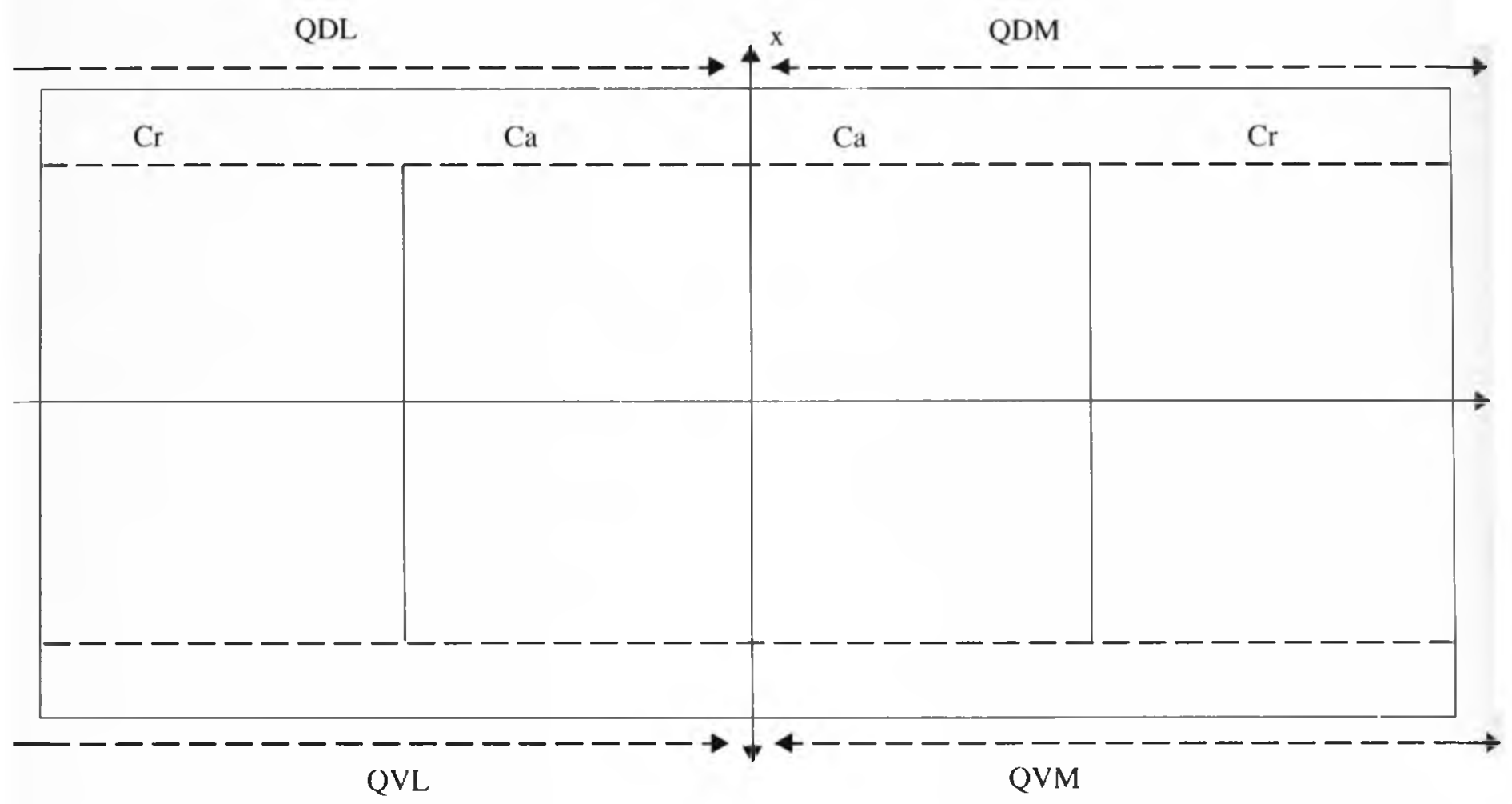

Quadro 1 - Desenho esquemático das preparaçōes, com divisāo em quadrantes do testículo.

TESTÍCULO ESQUERDO

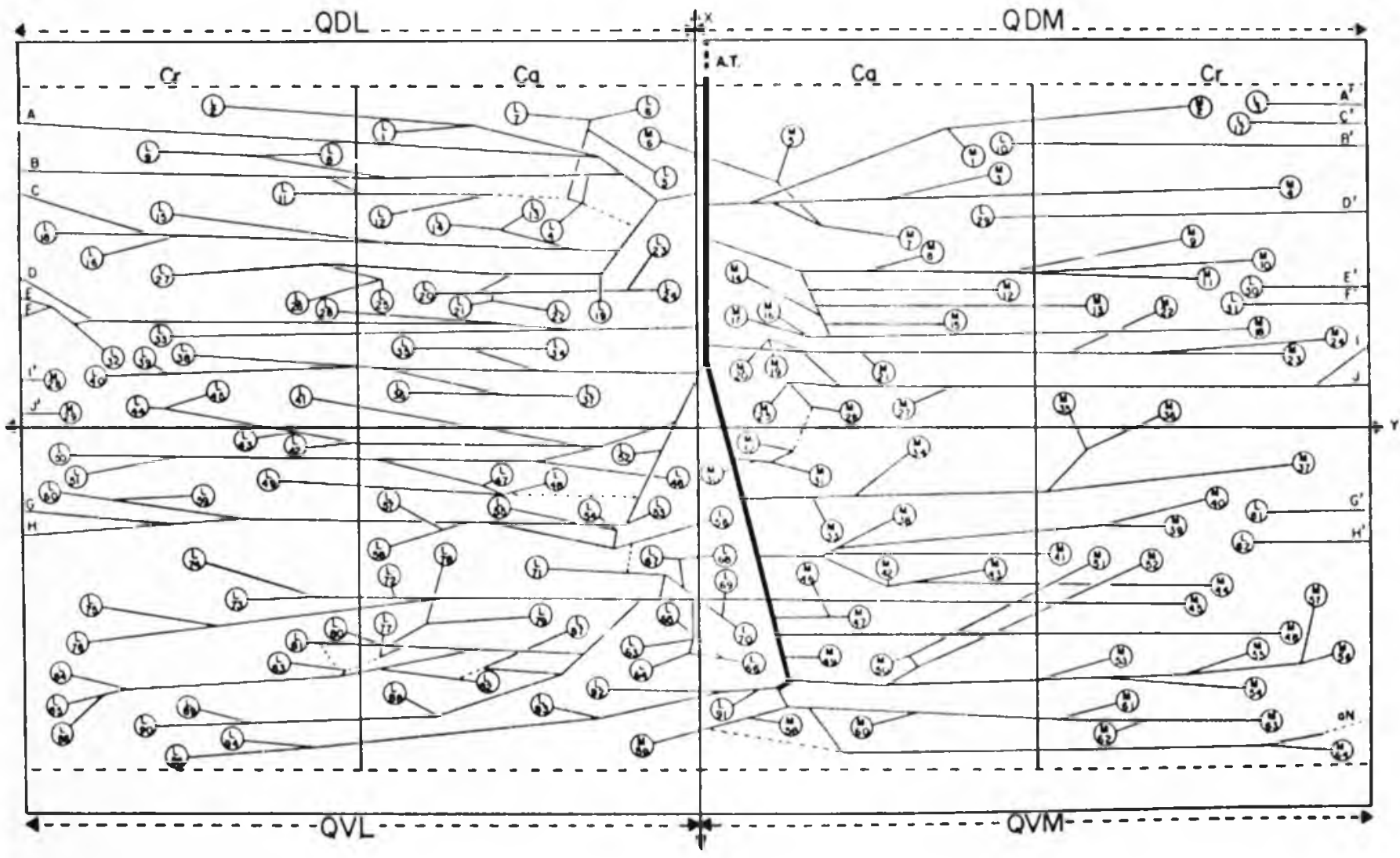

OBS- $19 E$

QDM - Quadrante dorsomedial / QDL - Quadrante dorsolateral / QVM-Quadrante ventromedial / QVL-Quadrante ventrolateral / Cr-Cranial / Ca-Caudal / a.T.-Artéria Testicular / r.M.-Ramo Medial / r.L.-Ramo Lateral/r.D.-Ramo Dorsal/r.y.-Ramo Ventral/r. A.-Ramo Adicional/ M-Vaso Penetrante Medial / I.-Vaso Penetrante Lateral / aN-Anastomose / D-Direito / F-F.squerdo / A-A" até I-I" - indicaçäo da continuidade dos vasos dos quadrantes craniais laterais para os mediais ou vice-versa. / _ _ anastomoses 